\title{
Bioactive Factors in Breast Milk: The Impact on Nutritional Programming of Childhood Obesity
}

\author{
Fei Xiong ${ }^{1,2}$ and Meng Mao*1 \\ ${ }^{1}$ Department of Pediatrics, West China Second University Hospital, Sichuan University, China \\ ${ }^{2}$ Key Laboratory of Birth Defects and Related Diseases of Women and Children, Sichuan University, China
}

Received: December 12, 2017; Published: December 20, 2017

*Corresponding author: Meng Mao, Department of Pediatrics, West China Second University Hospital, Sichuan University, Chengdu, Sichuan, 610041, China

\section{Abstract}

The concept of "nutritional programming" has led us to realize that early nutrition and metabolism have a very important impact on long-term health. There is a certain correlation between the growth of fetal and childhood and the occurrence of metabolic diseases in adults, such as obesity, type 2 diabetes, hypertension, and cardiovascular disease. Obesity is the most common metabolic disease whose prevalence is increasing worldwide. Nutrition in early stages of growth may be essential in the development of obesity in adulthood. Human milk is the most natural and ideal food for the baby. Moreover, breast-feeding appears to be associated with a lower risk of obesity than formula feeding. Human milk contains bioactive components, such as leptin, adiponectin, ghrelin, which are involved in food intake regulation and energy balance. These bioactive factors may contribute to childhood growth. In this review, we discuss bioactive factors contained in human milk and their potential protective effect on subsequent obesity.

Keywords : Breastfeeding; Obesity; Bioactive factors

Abbreviations: BMI: Body Mass Index; BF: Breast-Fed; SGA: Small for Gestational Age; AGA; Appropriate for Gestational Age; LGA: Large for Gestational Age; IGL: Insulin Like Growth Factor

\section{Introduction}

Obesity is considered the most common chronic metabolic disease associated co morbidities such as diabetes mellitus and hypertension [1]. The public health problem results primarily from an energy imbalance whereby dietary energy intake exceeds energy expenditure. The dynamics of this imbalance are complex, especially the appetite regulation are not fully understood [2]. Nutrition in early stages of growth may be essential in the development of obesity in adulthood, supporting the concept of "nutritional programming" $[3,4]$. However, the mechanisms that link nutrition with long-term obesity risk are not well defined. Several systematic reviews have linked breast milk intake with a protective effect against obesity and other metabolic diseases $[5,6]$. Breastfeeding may play an important role in this "nutritional programming". Human milk is a source of various growth bioactive factors, namely leptin, adiponectin, ghrelin, resistin and obestatin, which are involved in food intake regulation and energy balance. Some study referred the preventing function due to these bioactive factors' roles other than nutrition [7,8]. In this review, we talk about bioactive factors contained in human milk and their potential protective effect on obesity.

\section{Breast-Feeding and Childhood Obesity}

The rapid growth velocity during the early postnatal period ,especially 0 3 months, have been associated with an increase in the number of adipocytes, a higher ratio of fat mass to lean mass, a greater central fat deposition and insulin resistance and consequently an increased risk of metabolic syndrome, namely obesity, type 2 diabetes et al. Some systematic reviews confirmed that the link between greater growth acceleration and later increased risk of obesity $[9,10]$. As we known, formula feeding is associated with a greater weight and length gain after birth, according to the breast feeding. Various hypotheses have been proposed to explain how breastfeeding protects against faster weight gain and consequently against later obesity [11]. Breastfeeding babies can self-control the amount of milk they consume, and so they may learn to self-regulate their energy intake better than FF infants [12].

Furthermore, the different of the nutrient composition is an important factor determining a higher risk of later obesity. Another study showed that the mother's pre pregnant BMI, duration of 
breast-feeding and timing of complementary food introduction are associated with infant weight gain from birth to 1 year of life [13] The protective role of breast milk may be attributable not only to its nutritional composition but also to many bioactive factors. These bioactive factors in human milk, such as Leptin, adiponectin, ghrelin that may control nutrient use, protect infants from pathogens and play a role in regulating metabolic pathways [14].

\section{Hormones In Mother's Milk}

I. Leptin: Leptin can be produced by mammary epithelial cells. It exerts an orexigenic effect by signaling satiety and decreasing the sensation of hunger [15]. Breast milk leptin level is higher in colostrum than in transitional milk and is decreased during the first 180 days, showing a significant inverse relation with the ongoing days of lactation [16]. Schuster et al. and Fields et al. $[17,18]$ demonstrated that leptin concentration in milk had been positively correlated with circulating levels of leptin and maternal BMI, suggesting that BF infants nursed by overweight/ obese mothers might be exposed to higher amounts of leptin than infants nursed by lean mothers. Although the mechanisms are unknown, a higher concentration of circulating leptin has been found in infants fed breast milk than in infants fed formula [19]. Maybe the higher level of breast milk leptin could regulate the appetite and exert a long-term effect on energy balance and body weight regulation. Dundar et al. [20] found that SGA infants grew more rapidly during the first postnatal 15 days than AGA and LGA infants, and that human milk leptin levels were significantly lower in the SGA group. However, there is some inconsistent view. Wang et al. [21] found that the leptin level of human milk showed no significant difference between preterm groups with term group, had no correlation with weight, length at $42^{\text {nd }}$ day.

II. Adiponectin: In humans, adiponectin regulates lipid and glucose metabolism, improves insulin sensitivity, inversely related to the degree of adiposity and inhibits hepatic glucose production [8]. It is regulated by factors such as IGF-1 that stimulates its gene expression and secretion [22]. In 2006, it was the first time to report the presence of immuno reactive adiponectin in human breast milk. The authors also found the adiponectin levels in human milk were significantly higher than leptin levels, and decreased with the duration of lactation [23].

This adipokine secreted in human milk can cross the intestinal barrier and may modify infant metabolism. The levels of this hormone in human milk correlate positively with the serum level and inversely with infant weight and anthropometry during the first months of life [14,24]. Andreas et al reported that premature newborns have a lower concentration of adiponectin than term infants [25]. In the study of obese mothers, although serum adiponectin levels were low in obese mothers, their colostrum exhibited high levels of this hormone. Maternal BMI was positively associated with serum adipokine levels and negatively correlated with colostrum adipokine levels [26]. The offspring of obese mothers has the occurrence risk of metabolic syndrome, breast-feeding is a protective method.
III. Ghrelin: Ghrelin is also produced in the mammary gland, it can influence glucose metabolism, energy balance, gastrointestinal motility, gastric acid secretion, and cardiovascular and immune system function [27]. It can stimulate food intake in rats and humans. 64 by acting primarily on the accurate nucleus of the hypothalamus [28]. In fact, ghrelin occurs in both term and preterm human breast milk, the level is higher in breast milk than in plasma its levels, higher in whole milk than in skim milk [29]. The ghrelin level increases gradually in colostrum, and in transitional and mature milk [30]. Cesur et al. [31] reported that active ghrelin level in breast milk at the $4^{\text {th }}$ month of lactation significantly and positively correlated with weight gain of the infants. In new borns' levels of ghrelin were higher in SGA babies than in AGA babies. Reduced ghrelin suppression and higher postprandial ghrelin levels in SGA infants could result in a sustained orexigenic drive and could contribute to postprandial catch-up growth in these infants [32].

Savino et al. [33] observed significantly higher serum ghrelin levels in formula-fed compared to breast-fed infants. They suggested that formula fed infants received a higher amount of ghrelin, thus it was possible that they had a greater feeding stimulus than breast-fed infants and this correlates positively with a greater infant weight gain, possibly with an influence on the growth of the childhood. The resist in human breast milk was first identified in 2008 where the levels in milk decrease throughout lactation [14]. Its physiologic role in humans is still under debate and very little is known in children. Resist in has been shown to be associated with insulin resistance in obese mice [34]. It suggested that resist in could be involved in appetite regulation and in the metabolic development of infants. Moreover, it was advanced that it plays a role in controlling bodyweight through effective regulation of adipogenesis by negative feedback. However, in humans, the role of resist in fetal and infantile growth remains to be elucidated [35]. Other bioactive factors, such as apein, obestatin, nesfatin-1, can identified in breast milk. These substances may be regulate food intake and metabolism, however, the role of these bioactive factors in breast milk affecting the childhood growth is still not reach an agreement.

\section{Conclusion and Perspective}

Breast milk contains necessary nutrients and bioactive factors for infant health. The composition of breast milk varies according to stage of lactation and to the nutritional requirements of the infant. It is an advantage that is not comparable by formula feeding. The bioactive factors may represent the link between breast-feeding and protection against obesity in later life, which need large scale long term cohort study to confirm.

\section{References}

1. Patel MS, Srinivasan M (2010) Metabolic programming due to alterations in nutrition in the immediate postnatal period. J Nutr 140(3): 658-661.

2. Ross MG, Desai M (2014) Developmental programming of appetite/ satiety. Ann Nutr Metab 64(1): 36-44.

3. Vickers MH (2014) Developmental programming and trans generational transmission of obesity. Ann Nutr Metab 64(1): 26-34. 
4. Plagemann A, Harder T (2005) Breast feeding and the risk of obesity and related metabolic diseases in the child. Metab Syndr Relat Disord 3(3): 222-232.

5. Savino F, Liguori SA, Fissore MF, Oggero R (2010) Breast milk hormones and their protective effect on obesity. Int J Pediatr Endocrinol 2009(4): 327505 .

6. Vickers MH (2014) Developmental programming and trans generational transmission of obesity. Ann Nutr Metab 64(1): 26-34.

7. Hawkes J, Bryan DL, Gibson R (2004) Cells from mature human milk are capable of cytokine production following in vitro stimulation. Adv Exp Med Biol 554: 467-470.

8. Savino F, Liguori SA, Lupica MM (2010) Adipokines in breast milk and preterm infants. Early Hum Dev 86(Suppl 1): 77-80.

9. Monteiro PO, Victora CG (2005) Rapid growth in infancy and childhood and obesity in later life - a systematic review. Obes Rev 6(2): 143-154.

10. Baird J, Fisher D, Lucas P, Kleijnen J, Roberts H, et al. (2005) Being big or growing fast: systematic review of size and growth in infancy and later obesity. BMJ 331(7522): 929-931.

11. Singhal A, Lanigan J (2007) Breastfeeding, early growth and later obesity. Obes Rev 8(suppl1): 51-54.

12. Taveras EM, Scanlon KS, Birch L, Rifas-Shiman SL, Rich-Edwards JW, et al. (2004) Association of breastfeeding with maternal control of infant feeding at age 1 year. Pediatrics 114(5): e577-e583.

13. Baker JL, Michaelsen KF, Rasmussen KM, Sørensen TI (2004) Maternal prepregnant body mass index, duration of breastfeeding and timing of complementary food introduction are associated with infant weight gain. Am J Clin Nutr 80(6): 1579-1588.

14. Badillo-Suárez PA, Rodríguez-Cruz M, Nieves-Morales X (2017) Impact of Metabolic Hormones Secreted in Human Breast Milk on Nutritional Programming in Childhood Obesity. J Mammary Gland Biol Neoplasia 22(3): 171-191.

15. de Graaf C, Blom WA, Smeets PA, Stafleu A, Hendriks HF (2004) Biomarkers of satiation and satiety. Am J Clin Nutr 79(6): 946-961.

16. Ilcol YO, Hizli ZB, Ozkan T (2006) Leptin concentration in breast milk and its relationship to duration of lactation and hormonal status. Int Breastfeed J 1: 21.

17. Schuster S, Hechler C, Gebauer C, KiessW, Kratzsch J (2011) Leptin in maternal serum and breast milk: association with infants' body weight gain in a longitudinal study over 6 months of lactation. Pediatr Res 70(6): 633-637.

18. Fields D, George B, Williams M, Whitaker K, Allison D, et al. (2017) Associations between human breast milk hormones and adipocytokines and infant growth and body composition in the first 6 months of life. Pediatr Obes 12(suppl1): 78-85.

19. Houseknecht KL, McGuire MK, Portocarrero CP, McGuire MA, Beerman $\mathrm{K}$ (1997) Leptin is present in human milk and is related to maternal plasma leptin concentration and adiposity. Biochem Biophys Res Commun 240(3): 742-747.
20. Dundar NO, Anal O, Dundar B, Ozkan H, Caliskan S, et al. (2005) Longitudinal investigation of the relationship between breast milk leptin levels and growth in breast-fed infants. J Pediatr Endocrinol Metab 18(2): 181-187.

21. Han L, Li M, Yu X, Sun X, Wang D (2014) Assay of adiponectin, leptin, true insulin and ghrelin levels in preterm human milk, and its relationship with infants growth. Zhonghua Er Ke Za Zhi 52(7): 510-515.

22. Perry B, Wang Y (2012) Appetite regulation and weight control: the role of gut hormone. Nutr Diabetes 2(1): e26.

23. Martin LJ, Woo JG, Geraghty SR, Altaye M, Davidson BS, et al. (2006) Adiponectin is present in human milk and is associated with maternal factors. Am J Clin Nutr 83(5): 1106-1111.

24. Newburg DS, Woo JG, Morrow AL (2010) Characteristics and potential functions of human milk adiponectin. J Pediatr 156(2 Suppl): S41-S46.

25. Andreas N, Hyde M, Gale C, Parkinson J, Jeffries S, et al. (2014) Effect of Maternal Body Mass Index on Hormones in Breast Milk: A Systematic Review. Public Library Sci One 9(12): 1-25.

26. Fujimori M, França EL, Morais TC, Fiorin V, de Abreu LC, et al. (2017) Cytokine and adipokine are biofactors can act in blood and colostrum of obese mothers. Biofactors 43(2): 243-250.

27. Steculorum S, Collden G, Coupe B, Croizier S, Lockie S, et al. (2015) Neonatal ghrelin programs development of hypothalamic feeding circuits. J Clin Investig 125(2): 846-858.

28. Hellstrom PM (2009) Faces of ghrelin-research for the 21st century. Neurogastroenterol Motil 21(1): 2-5.

29. Kierson JA, Dimatteo DM, Locke RG, Mackley AB, Spear ML (2006) Ghrelin and cholecystokinin in term and preterm human breast milk. Acta Paediatr 95(8): 991-995.

30. Ilcol YO, Hizli B (2007) Active and total ghrelin concentrations increase in breast milk during lactation. Acta Paediatr 96(11): 1632-1639.

31. Cesur G, Ozguner F, Yilmaz N, Dundar B (2012) The relationship between ghrelin and adiponectin levels in breast milk and infant serum and growth of infants during early postnatal life. J Physiol Sci 62(3): 185190.

32. Chiesa C, Osborn JF, Haass C, Natale F, Spinelli M, et al. (2008) Ghrelin, leptin, IGF-1, IGFBP-3, and insulin concentrations at birth: is there a relationship with fetal growth and neonatal anthropometry? Clin Chem 54(3): 550-558.

33. Savino F, Petrucci E, Lupica MM, Nanni GE, Oggero R (2011) Assay of ghrelin concentration in infant formulas and breast milk. World J Gastroenterol 17(15): 1971-1975.

34. Savino F, Fissore MF, Liguori SA, Oggero R (2009) Can hormones contained in mothers' milk account for the beneficial effect of breastfeeding on obesity in children? Clin Endocrinol 71(6): 757-765.

35. Catlı G, Olgaç Dündar N, Dündar BN (2014) Adipokines in breast milk: an update. J Clin Res Pediatr Endocrinol 6(4): 192-201.

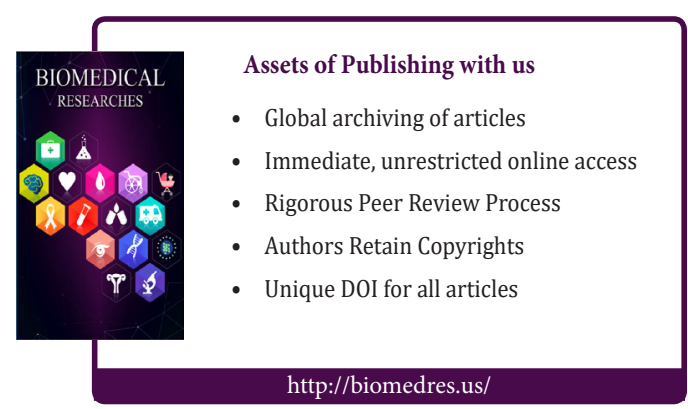

